\section{Terprenins, Novel Immunosuppressants Produced by Aspergillus candidus}

Sir:

During a screening program to find novel immunosuppressants from microbial fermentation products, we isolated terprenins ${ }^{1)}$ and terphenyllins ${ }^{2 \sim 4)}$ (Fig. 1 and 2) from the fermentation broth of Aspergillus candidus RF-5672 (FERM BP-5882), which was isolated from a soil sample collected on Shodo Island, Kagawa Prefecture, Japan. In this communication, we report the fermentation, isolation, structure elucidation and biological properties of these terprenins.

The activities of the terprenins found were evaluated with respect to proliferation of mouse spleen lymphocytes which had been stimulated with concanavalin A (Con A) and lipopolysaccharide (LPS). Spleen cells $\left(5 \times 10^{5}\right)$ taken from BDF1 mouse (Shizuoka Laboratory Animal Center, Hamamatsu, Japan), used as a responder, were mixed with stimulators Con A $(5 \mathrm{mcg} / \mathrm{ml})$ or LPS $(10$ $\mathrm{mcg} / \mathrm{ml})$. The responder cells were cultured with RPMI1640 medium (Gibco) containing 10\% fetal calf serum in 96-well micro-titer plates. Each well contained the responder cells, the stimulator (Con A or LPS) and test samples at a final volume of $0.2 \mathrm{ml}$. The plates were cultured at $37^{\circ} \mathrm{C}$ for 48 hours in $5 \% \mathrm{CO}_{2}$ in $100 \%$ humidified air. The inhibitory activities were determined by measuring the incorporation of $\left[{ }^{3} \mathrm{H}\right]$-thymidine into the cultured cells by liquid scintillation counting. The labeled reagent was pulsed 6 hours before the cell harvest.

A loopful of slant culture of strain Aspergillus candidus
RF-5672 was inoculated into 500-ml Erlenemyer flasks containing $100 \mathrm{ml}$ of seed medium consisting of $5.0 \%$ glucose, $5.0 \%$ corn steep liquor and $0.2 \% \mathrm{CaCO}_{3}$ in tap water, with the $\mathrm{pH}$ adjusted to 7.0 , and cultured on a rotary shaker $(220 \mathrm{rpm})$ at $25^{\circ} \mathrm{C}$ for 4 days. For the production of terprenins, 4-ml aliquots of each culture were transferred into twenty 500-ml Erlenmyer flasks, each containing $100 \mathrm{ml}$ of production medium consisting of $2.0 \%$ glycerine, $2.0 \%$ sucrose, $0.3 \%$ beef extract (Difco) and $0.2 \%$ yeast extract (Difco) in tap water, adjusted to $\mathrm{pH} 7.0$ and cultured on a rotary shaker $(180 \mathrm{rpm})$ at $23^{\circ} \mathrm{C}$ for 12 days.

The whole broth was subjected to filtration. The mycelial cake was extracted twice with acetone $(500 \mathrm{ml})$, which was then removed from the extract by evaporation. The mycelium extract and the filtrate were combined and then extracted twice with ethyl acetate $(500 \mathrm{ml})$ after adjusting the $\mathrm{pH}$ to 6.0 with $\mathrm{HCl}$. The organic layer was washed with water and evaporated to dryness under reduced pressure, giving an oily extract $(7.85 \mathrm{~g})$. The terprenins and terphenyllins were separated and purified by repeated silica gel column chromatography and reverse phase column chromatography from the extract according to Fig. 3.

The structures of terprenin (1), 3-methoxyterprenin (2) and 4 "-deoxyterprenin (3) were elucidated by ${ }^{1} \mathrm{H},{ }^{13} \mathrm{C}$ NMR and mass spectroscopy and confirmed by X-ray crystallographic analysis. The physico-chemical properties of the molecules are summarized in Table 1. The molecular formula was established as $\mathrm{C}_{25} \mathrm{H}_{26} \mathrm{O}_{6}$ for $\mathbf{1}$, $\mathrm{C}_{26} \mathrm{H}_{28} \mathrm{O}_{6}$ for 2 and $\mathrm{C}_{25} \mathrm{H}_{26} \mathrm{O}_{5}$ for 3 by HRSI-MS spectra and ${ }^{13} \mathrm{C}$ NMR spectra. In the UV spectra, for
Fig. 1. Structures of terprenins.

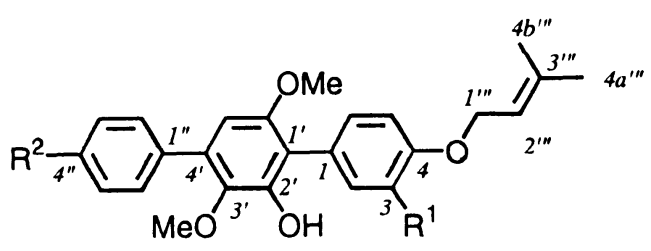

Fig. 2. Structures of terphenyllins.

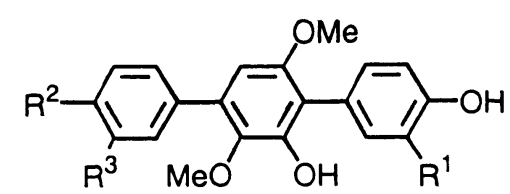

Terphenyllin (4)

$$
\text { : } \mathrm{R}^{1}=\mathrm{R}^{3}=\mathrm{H}, \mathrm{R}^{2}=\mathrm{OH}
$$

3-Hydroxyterphenyllin (5)

$\mathrm{R}^{1}=\mathrm{R}^{2}=\mathrm{OH}, \mathrm{R}^{3}=\mathrm{H}$

3,3"-Dihydroxyterphenyllin (6): $\mathrm{R}^{1}=\mathrm{R}^{2}=\mathrm{R}^{3}=\mathrm{OH}$

4"-Deoxyterphenyllin (7) $\quad: \mathrm{R}^{1}=\mathrm{R}^{2}=\mathrm{R}^{3}=\mathrm{H}$ 
Fig. 3. Isolation and purification of terprenins and terphenyllins.

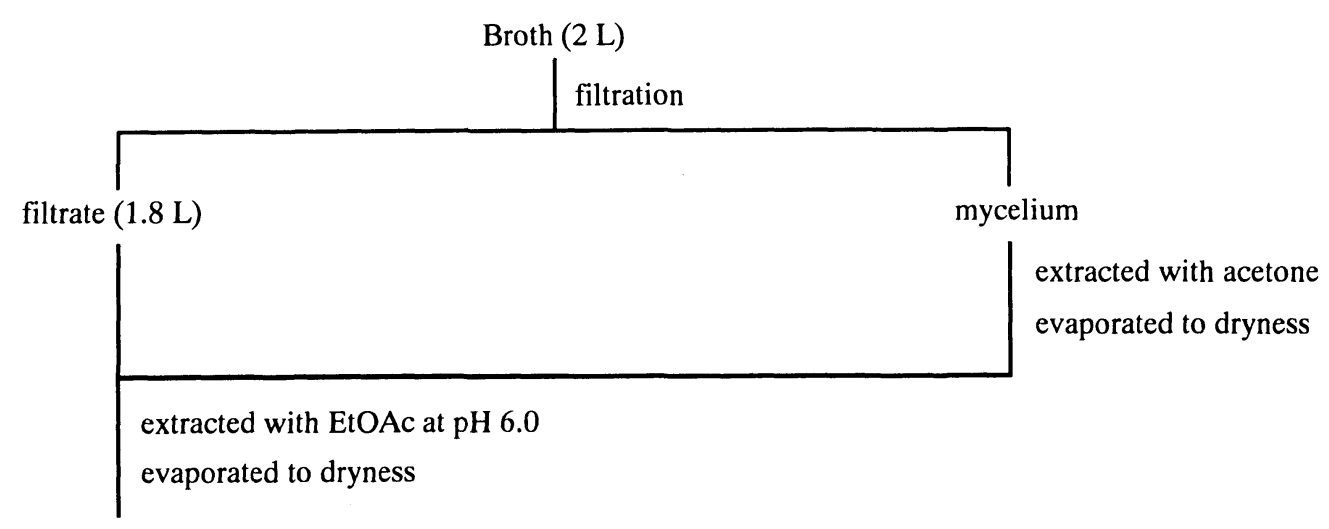

crude $(7.85 \mathrm{~g})$

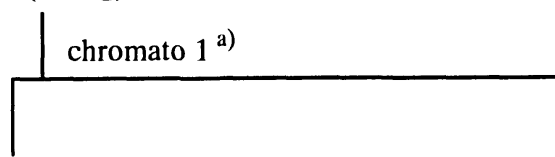

Fr. 1 $(0.657 \mathrm{~g})$

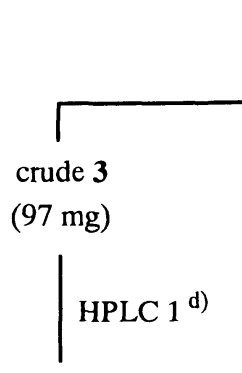

3

(70 mg)
$(2.7 \mathrm{mg})$ crude 2

(128 mg)

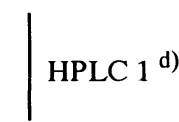

2 crude 1 \& 7

(117 mg)
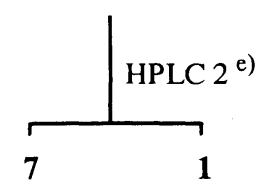

$(10 \mathrm{mg}) \quad(70 \mathrm{mg})$
Fr. 2

$(0.577 \mathrm{~g})$

chromato $3^{c)}$

HPLC $3{ }^{\text {f }}$

4

(90 mg)

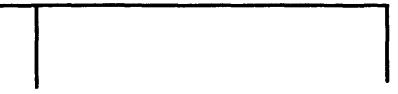

Fr. 3

$(1.6 \mathrm{~g})$

activated C recrystallization

$(1,000 \mathrm{mg})$
Fr. 4

(1.46 g) chromato $3^{\text {c) }}$

a) chromato $1\left(\mathrm{SiO}_{2}, 240 \mathrm{ml}, \mathrm{CHCl}_{3}, \mathrm{CHCl}_{3}: \mathrm{MeOH}=20: 1 \sim 20: 10\right)$

b) chromato $2\left(\mathrm{SiO}_{2}, 110 \mathrm{ml}\right.$, toluene $\left.: \mathrm{MeCN}=85: 15\right)$

c) chromato $3\left(\mathrm{SiO}_{2}, 90 \mathrm{ml}\right.$, toluene : $\left.\mathrm{MeCN}=80: 20\right)$

d) HPLC 1 (YMC GEL ODS-AM 120-S50, MeCN : $\mathrm{H}_{2} \mathrm{O}=7: 3$ )

e) HPLC 2 (YMC GEL ODS-AM 120-S50, MeCN : $\mathrm{H}_{2} \mathrm{O}=1: 1$ )

f) HPLC 3 (YMC GEL ODS-AM 120-S50, MeCN : $\mathrm{H}_{2} \mathrm{O}=4: 6$ )

example in terprenin (1), $\lambda_{\max }$ at $277 \mathrm{~nm}$ in the neutral solution shifted to longer wavelengths $(+20 \mathrm{~nm})$ in alkaline solution. This shift suggested that terprenins have a phenolic moiety in their structures. The ${ }^{1} \mathrm{H}$ and ${ }^{13} \mathrm{C}$ NMR spectral data of $\mathbf{1}, \mathbf{2}$ and $\mathbf{3}$ are summarized in Table 2. NMR analysis indicated terprenin (1) has an $O$-prenyl group $\left[\delta_{\mathrm{H}} 1.77,1.79(\right.$ each $3 \mathrm{H}), 4.63(2 \mathrm{H}), 5.52$ $(1 \mathrm{H})$ and $\delta_{\mathrm{C}} 18.31(\mathrm{q}), 26.00(\mathrm{q}), 66.18(\mathrm{t}), 121.44(\mathrm{~d})$, $137.50(\mathrm{~s})]$, two methoxyl groups $\left[\delta_{\mathrm{H}} 3.37,3.73\right.$ (each $3 \mathrm{H})$ and $\left.\delta_{\mathrm{C}} 56.04(\mathrm{q}), 60.67(\mathrm{q})\right]$, three phenolic hydroxyl groups $\left[\delta_{\mathrm{H}} 7.62,7.78,8.64\right]$ and three aromatic rings [an
ABX-type spin system on ring $\mathrm{A}\left(\delta_{\mathrm{H}} 6.83,6.96,6.92\right)$, a one proton system on ring $\mathrm{B}\left(\delta_{\mathrm{H}} 6.49\right)$ and an $\mathrm{A}_{2} \mathrm{~B}_{2}$-type spin system on ring $\left.C\left\{\delta_{\mathrm{H}} 6.94(2 \mathrm{H}), 7.54(2 \mathrm{H})\right\}\right]$. Long-range ${ }^{1} \mathrm{H}_{-}{ }^{13} \mathrm{C}$ correlations observed in the heteronuclear multiple-bond correlation (HMBC) spectrum of terprenin (1) suggested the proposed structure shown in Fig. 4.

3-Methoxyterprenin (2) was similar to terprenin (1), but the phenolic $\mathrm{OH}$ of the ring A was replaced by $\mathrm{OMe}$ $\left[\delta_{\mathrm{H}} 3.80(3 \mathrm{H}), \delta_{\mathrm{C}} 56.13(\mathrm{q})\right] .4^{\prime \prime}$-Deoxyterprenin (3) was also similar to terprenin (1), but the phenolic $\mathrm{OH}$ of ring 
Table 1. Physico-chemical properties of terprenins.

\begin{tabular}{|c|c|c|c|}
\hline & Terprenin (1) & 3-Methoxyterprenin (2) & 4"-Deoxyterprenin (3) \\
\hline Appearance & colorless prisms & white powder & white powder \\
\hline$M P^{\circ} \mathrm{C}$ & $155.5 \sim 156$ & - & - \\
\hline Molecular formula & $\mathrm{C}_{25} \mathrm{H}_{26} \mathrm{O}_{6}$ & $\mathrm{C}_{26} \mathrm{H}_{28} \mathrm{O}_{6}$ & $\mathrm{C}_{25} \mathrm{H}_{26} \mathrm{O}_{5}$ \\
\hline \multicolumn{4}{|l|}{ HRSI-MS } \\
\hline calcd & 422.1728 & 436.1884 & 406.1779 \\
\hline $\operatorname{obsd}(\mathbf{M})^{+}$ & 422.1730 & 436.1880 & 406.1780 \\
\hline \multicolumn{4}{|l|}{$\mathrm{UV} \lambda_{\max } \mathrm{nm}(\varepsilon)$} \\
\hline in $\mathrm{MeOH}$ & $230(\mathrm{sh}), 277(25,700)$ & $230(\mathrm{sh}), 278(25,300)$ & 225 (sh), $274(17,600)$ \\
\hline in $0.1 \mathrm{~N} \mathrm{NaOH}-\mathrm{MeOH}$ & $235(\mathrm{sh}), 297(26,200)$ & $235(\mathrm{sh}), 295(25,100)$ & $225(\mathrm{sh}), 255(\mathrm{sh}), 295(26,200)$ \\
\hline in $0.1 \mathrm{~N} \mathrm{HCl}-\mathrm{MeOH}$ & $230(\mathrm{sh}), 276(24,500)$ & $230(\mathrm{sh}), 278(24,500)$ & 225 (sh), $273(18,000)$ \\
\hline $\left.\operatorname{HPLC}(\min .)^{*}\right)$ & 5.6 & 7.4 & 13.5 \\
\hline
\end{tabular}

•) Column, YMC-Pack ODS-AM, AM-302 (4.6 i.d. x $150 \mathrm{~mm}$ ); flow rate, $1 \mathrm{ml} / \mathrm{min}$; detection, UV at $280 \mathrm{~nm}$; solvent, $\mathrm{CH}_{3} \mathrm{CN}: \mathrm{H}_{2} \mathrm{O}=55: 45$

Fig. 4. Long-range ${ }^{1} \mathrm{H}^{13} \mathrm{C}$ correlation of terprenin (1) by $\mathrm{HMBC}$ experiment.

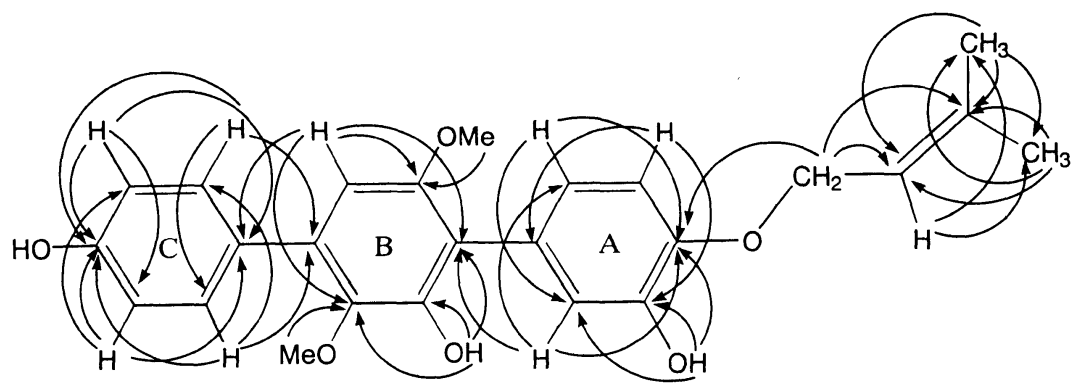

Fig. 5. The perspective view of terprenin (1) by X-ray crystallographic analysis.

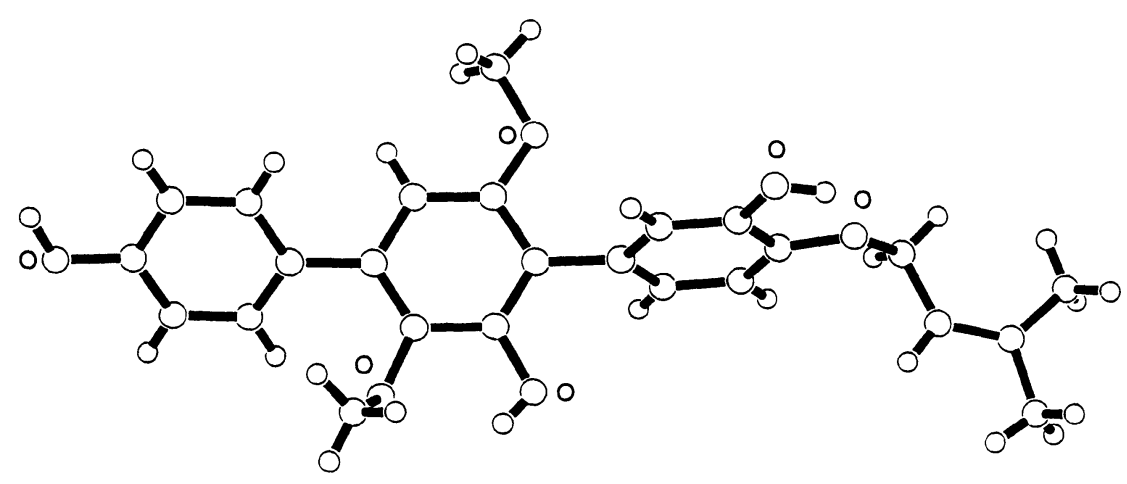


Table 2. ${ }^{1} \mathrm{H}$ and ${ }^{13} \mathrm{C}$ NMR spectral data of terprenins $\left(600 \mathrm{MHz}\right.$ in acetone- $\left.d_{6}\right)$.

\begin{tabular}{|c|c|c|c|c|c|c|}
\hline \multirow[b]{2}{*}{ Position } & \multicolumn{2}{|c|}{ Terprenin (1) } & \multicolumn{2}{|c|}{ 3-Methoxyterprenin (2) } & \multicolumn{2}{|c|}{ 4"-Deoxyterprenin (3) } \\
\hline & $\delta_{C}(\mathrm{ppm})$ & $\delta_{H}(\mathrm{ppm}, \mathrm{J}$ in $\mathrm{Hz})$ & $\delta_{C}(\mathrm{ppm})$ & $\delta_{\mathrm{H}}(\mathrm{ppm}, \mathrm{J}$ in $\mathrm{Hz})$ & $\delta_{C}(\mathrm{ppm})$ & $\delta_{H}(\mathrm{ppm}, \mathrm{J}$ in $\mathrm{Hz})$ \\
\hline 1 & $127.91(\mathrm{~s})$ & & $127.73(\mathrm{~s})$ & & $127.96(\mathrm{~s})$ & \\
\hline 2 & $118.97(d)$ & $6.92(\mathrm{~d}, 2.2)$ & $116.42(d)$ & $6.99(\mathrm{~d}, 2.0)$ & 119.07 (d) & 6.93(d, 2.0) \\
\hline 3 & $146.79(\mathrm{~s})$ & & $149.98(s)$ & & $147.07(\mathrm{~s})$ & \\
\hline $3-\mathrm{OH}$ & & 7.62 (br.s) & & & & $7.44(\mathrm{~s})$ \\
\hline 3-OMe & & & $56.13(q)$ & $3.80(s)$ & & \\
\hline 4 & $146.23(\mathrm{~s})$ & & $148.37(\mathrm{~s})$ & & $146.50(\mathrm{~s})$ & \\
\hline 5 & $112.75(d)$ & $6.96(\mathrm{~d}, 8.2)$ & $113.80(d)$ & $6.97(\mathrm{~d}, 8.3)$ & $113.10(d)$ & $6.97(d, 8.2)$ \\
\hline 6 & $123.15(d)$ & $6.83(\mathrm{dd}, 8.2 \& 2.2)$ & $124.37(\mathrm{~d})$ & $6.93(\mathrm{dd}, 8.3 \& 2.0)$ & $123.28(d)$ & $6.84(\mathrm{dd}, 8.2 \& 2.0)$ \\
\hline $1^{\prime}$ & $117.61(\mathrm{~s})$ & & $117.70(\mathrm{~s})$ & & $118.63(\mathrm{~s})$ & \\
\hline $2^{\prime}$ & 149.24 (s) & & $149.16(s)$ & & $149.40(\mathrm{~s})$ & \\
\hline $2^{\prime}-\mathrm{OH}$ & & $7.78(\mathrm{~s})$ & & $7.83(s)$ & & $7.65(\mathrm{~s})$ \\
\hline $3^{\prime}$ & $140.04(s)$ & & $140.17(\mathrm{~s})$ & & $140.46(s)$ & \\
\hline 3'-OMe & $60.67(q)$ & $3.37(\mathrm{~s})$ & $60.65(q)$ & $3.38(\mathrm{~s})$ & $61.04(q)$ & $3.38(\mathrm{~s})$ \\
\hline $4^{\prime}$ & $133.54(\mathrm{~s})$ & & $133.66(\mathrm{~s})$ & & $133.85(\mathrm{~s})$ & \\
\hline $5^{\prime}$ & $103.85(d)$ & $6.49(\mathrm{~s})$ & 104.17 (d) & $6.50(\mathrm{~s})$ & $104.45(d)$ & $6.52(\mathrm{~s})$ \\
\hline $6^{\prime}$ & $154.51(\mathrm{~s})$ & & $154.52(\mathrm{~s})$ & & $154.78(\mathrm{~s})$ & \\
\hline 6'-OMe & $56.04(q)$ & $3.73(\mathrm{~s})$ & $56.16(q)$ & $3.74(\mathrm{~s})$ & $56.26(q)$ & $3.73(\mathrm{~s})$ \\
\hline $1^{\prime \prime}$ & $130.48(s)$ & & $130.50(\mathrm{~s})$ & & $139.65(\mathrm{~s})$ & \\
\hline $2 "$ & $130.85(\mathrm{~d})$ & $7.54(\mathrm{~m})$ & 130.79 (d) & $7.54(\mathrm{~m})$ & $129.84(d)$ & $7.66(\mathrm{~m})$ \\
\hline 3" & $116.06(d)$ & $6.94(\mathrm{~m})$ & $116.06(d)$ & $6.95(\mathrm{~m})$ & $129.35(d)$ & $7.46(\mathrm{~m})$ \\
\hline $4^{\prime \prime}$ & $157.79(\mathrm{~s})$ & & $157.80(\mathrm{~s})$ & & $128.25(d)$ & $7.35(\mathrm{~m})$ \\
\hline $4 "-\mathrm{OH}$ & & 8.64 (br. s) & & $8.65(\mathrm{~s})$ & & \\
\hline $5^{\prime \prime}$ & $116.06(d)$ & $6.94(\mathrm{~m})$ & $116.06(d)$ & $6.95(\mathrm{~m})$ & $129.35(\mathrm{~d})$ & $7.46(\mathrm{~m})$ \\
\hline 6" & $130.85(d)$ & $7.54(\mathrm{~m})$ & 130.79 (d) & $7.54(\mathrm{~m})$ & 129.84 (d) & $7.66(\mathrm{~m})$ \\
\hline $1^{\prime \prime \prime}$ & $66.18(t)$ & 4.63 (br. d, 6.6) & $66.18(t)$ & 4.59 (d-like, 6.7) & $66.44(t)$ & $4.63(\mathrm{~m})$ \\
\hline $2^{\prime \prime \prime}$ & $121.44(d)$ & $5.52(\mathrm{~m})$ & $121.63(d)$ & $5.53(\mathrm{~m})$ & $121.56(d)$ & $5.53(\mathrm{~m})$ \\
\hline 3'" & $137.50(\mathrm{~s})$ & & $137.40(\mathrm{~s})$ & & $137.70(\mathrm{~s})$ & \\
\hline 4a'" & $26.00(q)$ & $1.79(\mathrm{~m})$ & $25.84(q)$ & 1.79 (s-like) & $26.00(q)$ & 1.78 (s-like) \\
\hline $4 b^{\prime \prime \prime}$ & $18.31(\mathrm{q})$ & $1.77(\mathrm{~m})$ & $18.19(q)$ & 1.77 (s-like) & $18.34(q)$ & 1.77 (s-like) \\
\hline
\end{tabular}

C was replaced by a proton $\left[\delta_{\mathrm{H}} 7.35(1 \mathrm{H}), \delta_{\mathrm{C}} 128.25\right.$ (d)]. These structures ( 2 and $\mathbf{3}$ ) were confirmed by HMBC and NOESY experiments.

The proposed structure of terprenin (1) was confirmed also by X-ray crystallographic analysis (Fig. 5). Crystals suitable for X-ray analysis were grown from solvent mixtures of $n$-hexane and ethyl acetate. The crystal data are as follows: monoclinic, space group $\mathrm{P} 21 / \mathrm{n}$, $\mathrm{a}=12.097(1) \AA, \quad \mathrm{b}=13.840(2) \AA, \quad \mathrm{c}=15.534(1) \AA$, $\beta=107.07(1)^{\circ}, V=2486.1(4) \AA^{3}, Z=4$.

Terprenins possessed very strong proliferation against mouse spleen lymphocytes stimulated with Con A and LPS. The $\mathrm{IC}_{50}$ values of terprenin (1), 3-methoxyterprenin (2) and 4"-deoxyterprenin (3) were calculated 
Table 3. Effect of terprenins against Con A-induced proliferation.

\begin{tabular}{|c|c|c|c|c|c|c|c|c|c|}
\hline \multirow[b]{2}{*}{$(\mathrm{ng} / \mathrm{ml})$} & \multirow{2}{*}{$\begin{array}{l}1 \\
1 \\
1 \\
1 \\
1\end{array}$} & \multicolumn{2}{|c|}{ Terprenin (1) } & $\begin{array}{c}T \\
\vdots \\
\end{array}$ & \multicolumn{2}{|c|}{ 3-Methoxyterprenin (2) } & ! & \multicolumn{2}{|c|}{ 4"-Deoxyterprenin (3) } \\
\hline & & $\begin{array}{c}\text { Radioactivity } \\
\qquad \mathrm{cpm} \pm \mathrm{SD}\end{array}$ & $\begin{array}{c}\text { Inhibition } \\
\%\end{array}$ & i & $\begin{array}{c}\text { Radioactivity } \\
\text { cpm } \pm S D\end{array}$ & $\begin{array}{c}\text { Inhibition } \\
\%\end{array}$ & $\vdots$ & $\begin{array}{c}\text { Radioactivity } \\
\text { cpm } \pm S D\end{array}$ & $\begin{array}{c}\text { Inhibition } \\
\%\end{array}$ \\
\hline - Con A & ! & $3,440 \pm 568$ & 100 & $\begin{array}{l}1 \\
\vdots\end{array}$ & $3,440 \pm 568$ & 100 & 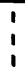 & $3,440 \pm 568$ & 100 \\
\hline 0 & ! & $277,061 \pm 7,118$ & 0 & : & $277,061 \pm 7,118$ & 0 & ! & $277,061 \pm 7,118$ & 0 \\
\hline 0.25 & ! & $292,470 \pm 542$ & -5.6 & i & $285,408 \pm 7,252$ & -3.1 & 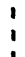 & $281,904 \pm 6,522$ & -1.8 \\
\hline 0.98 & ! & $210,046 \pm 3,288$ & 24.5 & i & $266,173 \pm 6,208$ & 4.0 & i & $281,371 \pm 10,119$ & -1.6 \\
\hline 3.91 & 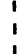 & $56,871 \pm 1,554$ & 80.5 & 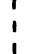 & $101,504 \pm 1,326$ & 64.2 & i & $191,575 \pm 6,969$ & 30.9 \\
\hline 15.60 & ! & $11,366 \pm 372$ & 97.1 & i & $20,510 \pm 287$ & 93.8 & 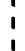 & $41,660 \pm 531$ & 86.0 \\
\hline 62.50 & i & $6,411 \pm 246$ & 98.9 & i & $7,755 \pm 624$ & 98.4 & ; & $11,793 \pm 235$ & 96.9 \\
\hline 250.00 & 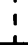 & $6,404 \pm 403$ & 98.9 & $i$ & $7,522 \pm 485$ & 98.5 & ' & $8,233 \pm 254$ & 98.2 \\
\hline $\mathrm{IC}_{50}(\mathrm{ng} / \mathrm{ml})$ & & 1.2 & & ! & 2.0 & & & 5.6 & \\
\hline
\end{tabular}

Table 4. Effect of terprenins against LPS-induced proliferation.

\begin{tabular}{|c|c|c|c|c|c|c|c|c|}
\hline \multirow[b]{2}{*}{$(\mathrm{ng} / \mathrm{ml})$} & \multicolumn{2}{|c|}{ Terprenin (1) } & I & \multicolumn{2}{|c|}{ 3-Methoxyterprenin (2) } & ! & \multicolumn{2}{|c|}{ 4"-Deoxyterprenin (3) } \\
\hline & $\begin{array}{l}\text { Radioactivity } \\
\qquad \mathrm{cpm} \pm \mathrm{SD}\end{array}$ & $\begin{array}{c}\text { Inhibition } \\
\%\end{array}$ & i & $\begin{array}{c}\text { Radioactivity } \\
\text { cpm } \pm S D\end{array}$ & $\begin{array}{c}\text { Inhibition } \\
\%\end{array}$ & : & $\begin{array}{c}\text { Radioactivity } \\
\text { cpm } \pm S D\end{array}$ & $\begin{array}{c}\text { Inhibition } \\
\%\end{array}$ \\
\hline - LPS & $2,939 \pm 167$ & 100 & ! & $2,939 \pm 167$ & 100 & ! & $2,939 \pm 167$ & 100 \\
\hline 0 & $153,851 \pm 5,649$ & 0 & & $153,851 \pm 5,649$ & 0 & ! & $153,851 \pm 5,649$ & 0 \\
\hline 0.98 & $153,396 \pm 6,123$ & 0.3 & 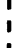 & $184,366 \pm 10,625$ & -20.2 & i & $208,023 \pm 8,941$ & -35.9 \\
\hline 3.91 & $88,405 \pm 10,394$ & 43.4 & & $128,436 \pm 4,167$ & 16.8 & ' & $132,834 \pm 5,106$ & 13.9 \\
\hline 15.60 & $32,548 \pm 315$ & 80.4 & 1 & $46,765 \pm 2,209$ & 71.0 & ' & $78,686 \pm 4,135$ & 49.8 \\
\hline 62.50 & $16,070 \pm 944$ & 91.3 & & $22,961 \pm 1,187$ & 86.7 & ! & $39,824 \pm 651$ & 75.6 \\
\hline 250.00 & $15,046 \pm 344$ & 92.0 & 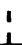 & $14,962 \pm 866$ & 92.0 & 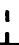 & $22,312 \pm 1,122$ & 87.2 \\
\hline $\mathrm{lC}_{50}(\mathrm{ng} / \mathrm{ml})$ & 4.5 & & 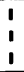 & 8.0 & & & 15 & \\
\hline
\end{tabular}

as $1.2,2.0$ and $5.6 \mathrm{ng} / \mathrm{ml}$ against Con A-induced proliferation and $4.5,8.0$ and $15.6 \mathrm{ng} / \mathrm{ml}$ against LPSinduced proliferation. These values are summarized in Table 3 and 4 . Terprenins had no antimicrobial activity against bacteria and fungi.

To further clarify the biological properties, we established the route of chemical conversion to terprenin (1) from 3-hydroxyterphenyllin (5). The route included a prenylation step (prenyl bromide, $\mathrm{K}_{2} \mathrm{CO}_{3}$ and acetone) and a separation step (ODS column, $\mathrm{MeCN}: \mathrm{H}_{2} \mathrm{O}=$ $1: 1)$ which separated the 4-O-prenylated product (=terprenin) and the 3-O-prenylated by-product. M. OHTANI, K. KaWADA and their co-workers at Shionogi
Research Laboratories have recently finished the first total synthesis of terprenin ${ }^{5}$.

We isolated three new $O$-prenylated para-terphenyl compounds, terprenin (1), 3-methoxyterprenin (2) and 4"-deoxyterprenin (3), which possess strong immunosuppressive activities in vitro. No such activity was found for four known para-terphenyl compounds, terphenyllin (4), 3-hydroxyterphenyllin (5), 3,3"-dihydroxyterphenyllin (6) and 4"-deoxyterphenyllin (7).

The action mechanisms of the active terprenins are now under study. 
TOSHIYUKI KAMIGAUCHI* RYUJI SAKAZAKI

KazUO NagaSHima

YOSHIMI KaWAMURA

YUKIO YASUDA

Keisuke Matsushima

HIROYOSHI TANI

YaSUO TAKAHASHI

KIKUO ISHII

RYUJi SuZuki

Kenzo KoIZUMI

HIROSHI NAKAI

YUJI IKENISHI

YoshiHIRO TERUI

Shionogi Research Laboratories, Shionogi \& Co., Ltd., Fukushima-ku, Osaka 553-0002, Japan

(Received December 8, 1997)

\section{References}

1) Kamigauchi, T. \& R. Suzuki (Shionogi \& Co., Ltd.): Novel terphenyl compounds and medicinal composition. PCT WO97/39999, October 30, 1997

2) Takahashi, C.; K. Yoshihira, S. Natori \& M. Umeda: The structures of toxic metabolites of Aspergillus candidus. I. The compounds A and E, cytotoxic p-terphenyls. Chem. Pharm. Bull. 24(4): 613 620, 1976

3) Kurobane, I.; L. C. Vining, A. G. Mcinnes \& D. G. SMiтh: 3-Hydroxyterphenyllin, a new metabolite of Aspergillus candidus structure elucidation by ${ }^{1} \mathrm{H}$ and ${ }^{13} \mathrm{C}$ nuclear magnetic resonance spectroscopy. J. Antibiotics 32(6): $559 \sim 564,1979$

4) Kobayashi, A.; A. Takemoto, K. Koshimizu \& K. KAWAZU: $p$-Terphenyls with cytotoxic activity toward sea urchin embryos. Agric. Biol. Chem. 49(3): 867 868, 1985

5) Kawada, K.; A. Arimura, T. Tsuri, M. Fuji, T. Komurasaki, S. Yonezawa, A. Kugimiya, N. Haga, M. Inagaki, T. Nakatani, Y. Tamura, S. Takechi, T. TAISHI, J. Kishino \& M. OHTANI: Total synthesis of terprenin, a highly potent and novel immunoglobulin $\mathrm{E}$ antibody suppressant. Angew. Chem. in press 